\title{
Efficacy of brief behavioral counselling by allied health professionals to promote physical activity in people with peripheral arterial disease (BIPP): study protocol for a multi-center randomized controlled trial
}

Nicola W. Burton ${ }^{1}$, Zanfina Ademi ${ }^{2,3}$, Stuart Best ${ }^{4}$, Maria A. Fiatarone Singh ${ }^{5}$, Jason S. Jenkins ${ }^{6}$, Kenny D. Lawson ${ }^{7,8}$, Anthony S. Leicht ${ }^{9}$, Yorgi Mavros ${ }^{10}$, Yian Noble ${ }^{10}$, Paul Norman ${ }^{11}$, Richard Norman ${ }^{12}$, Belinda J. Parmenter ${ }^{13}$, Jenna Pinchbeck ${ }^{4}$, Christopher M. Reid ${ }^{12,14}$, Sophie E. Rowbotham ${ }^{15,16}$, Lisan Yip ${ }^{4}$ and Jonathan Golledge ${ }^{4,17^{*}}$

\begin{abstract}
Background: Physical activity is recommended for people with peripheral arterial disease (PAD), and can improve walking capacity and quality of life; and reduce pain, requirement for surgery and cardiovascular events. This trial will assess the efficacy of a brief behavioral counselling intervention delivered by allied health professionals to improve physical activity in people with PAD.

Methods: This is a multi-center randomised controlled trial in four cities across Australia. Participants $(N=200)$ will be recruited from specialist vascular clinics, general practitioners and research databases and randomised to either the control or intervention group. Both groups will receive usual medical care, a written PAD management information sheet including advice to walk, and four individualised contacts from a protocol-trained allied health professional over 3 months (weeks 1, 2, 6, 12). The control group will receive four 15-min telephone calls with general discussion about PAD symptoms and health and wellbeing. The intervention group will receive behavioral counselling via two 1-h face-to-face sessions and two 15-min telephone calls. The counselling is based on the 5A framework and will promote interval walking for $3 \times 40 \mathrm{~min} /$ week. Assessments will be conducted at baseline, and 4, 12 and 24 months by staff blinded to participant allocation. Objectively assessed outcomes include physical activity (primary), sedentary behavior, lower limb body function, walking capacity, cardiorespiratory fitness, event-based claudication index, vascular interventions, clinical events, cardiovascular function, circulating markers, and anthropometric measures. Self-reported outcomes include physical activity and sedentary behavior, walking ability, pain severity, and health-related quality of life. Data will be analysed using an intention-to-treat approach. An economic evaluation will assess whether embedding the intervention into routine care would likely be value for money. A cost-effectiveness analysis will estimate change in cost per change in activity indicators due to the intervention, and a cost-utility analysis will assess change in cost per quality-adjusted life year. A full uncertainty analysis will be undertaken, including a value of information analysis, to evaluate the economic case for further research.

(Continued on next page)
\end{abstract}

\footnotetext{
* Correspondence: Jonathan.Golledge@jcu.edu.au

${ }^{4}$ Queensland Research Centre for Peripheral Vascular Disease; College of

Medicine and Dentistry, James Cook University, Townsville, QLD 4811, Australia

${ }^{17}$ Department of Vascular and Endovascular Surgery, The Townsville Hospital,

Townsville, QLD 4811, Australia

Full list of author information is available at the end of the article
} 
(Continued from previous page)

Discussion: This trial will evaluate the efficacy and cost-effectiveness of a brief behavioral counselling intervention for a common cardiovascular disease with significant burden.

Trial registration: ACTRN 12614000592640 Australian New Zealand Clinical Trials Registry. Registration Date 4 June 2014.

Keywords: Exercise, Physical inactivity, Intermittent claudication, Cardiovascular disease, Health counselling, Quality of life, Cost effectiveness, Biomarkers, Multi-disciplinary, Behavioral health

\section{Background}

Peripheral artery disease (PAD) is the narrowing or blockage of the lower limb arteries usually due to atherosclerosis and associated thrombosis [1]. This reduces blood flow to the limbs, with the most recognised symptom being leg pain that typically presents during walking, worsens with exertion, and is relieved by rest (intermittent claudication: IC). Maximal walking capacity in people with PAD is less than $50 \%$ of that in age-matched controls, and the functional limitations are similar to those seen in people with severe heart failure [2]. People with PAD and IC typically avoid walking and related activities, which constrains social and role functioning and activities of daily living, and precipitates poor quality of life and accelerated functional decline [3]. This, in turn, predisposes them to high health service utilisation and associated costs [4-11], and premature mortality [12]. As an indicator of systemic atherosclerosis, PAD is positively associated with other cardiovascular diseases, coronary events and stroke [13], with one meta-analysis suggesting a two-fold increased risk of mortality, cardiovascular mortality and major coronary events over 10 years [14]. Coronary heart and cerebrovascular events account for $\sim 65 \%$ of deaths in people with PAD, with a two year prospective study suggesting a $70 \%$ increased risk of cardiovascular events and an $80 \%$ increased risk of death compared to people with coronary artery disease alone [13].

PAD is a common chronic disease associated with ageing. Prevalence has been reported as $8.3 \%$ in people aged 60-69 years [6], $14.5 \%$ in those aged $>70$ years [15] and $40 \%$ of people aged $>80$ years [16]. The economic burden of PAD is substantial, as reflected by expensive vascular surgical interventions aimed at opening or bypassing blocked arteries, and recurring hospitalisations [7]. A total of USD $\$ 4.37$ billion was spent on PAD-related treatment in 2001, with $88 \%$ for inpatient care [17]. Prior lower limb revascularisation is associated with high hospitalisation costs and rates of subsequent procedures during the following 2 years [7]. Rates of surgical intervention are increasing dramatically: There was a $43 \%$ increase between 2000 and 2008 in Australia which far exceeds the estimated growth in the number of people with PAD [18]. Such interventions have limited durability, are expensive to perform and their value in providing sustained improvement in quality of life and reducing subsequent morbidity and mortality is not well established $[19,20]$. Given the high prevalence and burden of PAD, and the large expense and limited durability of surgical interventions, there is an urgent need to identify effective, affordable and sustainable alternative management options.

International guidelines for PAD management recommend exercise [21-24], and typically identify three 40-min sessions per week of interval walking at maximum tolerable speed (i.e., moderate pain level with rest periods) $[2,25]$. There is good evidence that supervised exercise is as effective as surgical procedures in improving walking ability in patients that have IC [20, 26, 27]. Supervised exercise programs have been shown to improve walking capacity, functional mobility, maximal walking distance, pain symptoms and health-related quality of life; and to reduce leg pain and cardiovascular risk [20, 28-32]. People with PAD who are physically active have a lower average annual functional decline than those who are inactive [33], and a significantly lower five-year mortality risk [12, 34]. Reduced daily sitting time is associated with a reduced decline in the six minute walk test [35], with improvements in walking capacity and physical functioning enhancing quality of life in people with PAD with IC and people without IC [36]. It is estimated that even small increases in physical activity for people with IC would significantly reduce all-cause mortality [12].

However, an international survey found only $30 \%$ of vascular surgeons had access to supervised exercise programmes for patients with PAD, and of those who did have access, many referred less than half their patients [37]. Patient travel to facility-based supervised exercise sessions is burdensome, and drop-out rates are often high [38-40]. Lack of patient and physician awareness of the role of supervised exercise, and patient barriers of transportation and time contribute to this being an underused treatment [37, 41, 42]. For those surgeons who do not have access to refer to supervised exercise programmes, the usual option is to give brief advice to do walking [37].

However, people with IC have condition-specific barriers to walking that constrain participation. IC is an intense, cramping pain in the lower limbs, and some patients 
believe this pain is harmful [43], and have low confidence in their walking ability $[44,45]$. Patients tend to be unclear about the causes and severity of PAD and the associated cardiovascular risk [43, 46, 47], and are unaware of walking recommendations and the mechanisms by which walking can improve PAD-specific and general health outcomes [43]. The surgical model of care can precipitate beliefs of an acute, treatable condition [43] and little personal control over health outcomes [44, 47]. Illness and treatment beliefs predict adherence to recommended levels of walking in people with PAD with very high accuracy [44]. A willingness to persist with activities given the concurrent pain, and a desire to be functionally competent, are also positively associated with physical mobility in people with PAD [45]. Adaptive self-management beliefs are therefore a key target to enable walking among people with PAD.

Behavioural counselling is a patient-centred approach that can promote adaptive illness- and exercise-related beliefs associated with sustained physical activity behaviour change in people with chronic conditions and cardiovascular risk $[48,49]$. Preliminary evidence indicates that counselling can improve physical activity in people with PAD. A program combining weekly cognitive behavioral counselling and walking sessions demonstrated significant improvements in accelerometer assessed physical activity (mean difference 114.7 activity units, $95 \%$ CI 12.82 to $216.5 ; P=.03$ ); 6-min walk distance (mean difference $53.5 \mathrm{~m}, 95 \%$ confidence interval [CI] 33.2 to 73.8 ; $P<.001$ ); Walking Impairment Questionnaire (WIQ) speed score (mean difference 10.4, 95 \% CI 3.4 to $17.4 ; P=0.004$ ) and distance score (mean difference 11.1, $95 \%$ CI 3.9 to $18.1 ; P=0.003$ ) [50]; and psychosocial indicators of satisfaction with physical functioning, pain acceptance and social functioning [51] relative to a health education control group. There were sustained improvements after 12 months in 6-min walk distance (mean difference $34.1 \mathrm{~m}$, $95 \%$ CI, 14.6 to $53.5 ; P<0.001$ ) and WIQ speed score (mean difference 8.8, $95 \%$ CI, 1.6 to $16.1 ; P<0.018$ ) [52]. However, this approach was resource intensive, with weekly sessions delivered over 6 months. A more brief counselling intervention, involving two 1-h sessions of individualised behavioral counselling and two follow up telephone calls by a psychologist, demonstrated a significant increase in pain-free walking distance and daily walking in the intervention versus the control group (usual care plus non-walking related conversation during home visits) after four months, with a mean difference of 1575 pedometer steps/day (95\% CI, 732 to 2419) [53]. At 24 months, the intervention group completed on average 1630 steps/day (95 \% CI, 495 to 2765) more than the control group, and significantly more participants in the control group had endovascular or open surgery than in the intervention group (OR $3.0995 \%$ CI 1.06 to $9.04 ; p=0.037$ ) [54]. However, as the counselling was delivered by a psychologist in patients' homes, the wide scale applicability of this approach is not known.

\section{Methods/Design}

\section{Aim and research questions}

The primary aim of this study is to test the efficacy of brief behavioral counselling, delivered by allied health professionals, to increase physical activity in people with PAD. Secondary aims are to examine the effect of the counselling on functional capacity, revascularisation rate, clinical events, and resource use; and to perform an economic evaluation of the counselling intervention compared to usual care. This fills an important gap in the literature on PAD clinical management, and will address the following questions

- can brief behavioural counselling be delivered by health professionals who are not qualified psychologists, following training?

- can the brief behavioural counselling be effectively delivered in a variety of different centres by different professionals?

- is delivery of the brief behavioural counselling in a health care setting effective in achieving sustained physical activity behaviour change?

\section{Design and setting}

This is a multicentre, prospective, randomised superiority trial with two parallel study groups: a control and an intervention group. The trial will be conducted in four cities across Australia. Study sites will include three specialist vascular management clinics (Townsville, Brisbane, Perth) and a University clinic (Sydney).

\section{Management}

The trial will be overseen by a Trial Steering Committee comprising senior vascular investigators from the centers involved; and experts in trial management, physical activity behavior change, health economics and exercise physiology. This will be the main policy and decision-making committee. Trial coordination will be facilitated by the National Health and Medical Research Council (NHMRC) funded National Centre for Research Excellence in Peripheral Arterial Disease (NCRE-PAD). An independent data management center (CCRE therapeutics, Monash University) will provide a computer generated randomisation list. A study coordinator located at James Cook University will organise activities across the participating sites; assisting with local ethics reports and communication with the local sites (including uniformity of collection and reporting of data, central randomisation, data storage and processing). The training, competency assessment, and supervision of allied health professionals who will deliver the intervention and control group protocol; and the intervention fidelity assessment, will be undertaken by a clinical and health psychologist on the investigatory team (NB). 


\section{Participants}

Participants will be recruited from specialist vascular management clinics at The Townsville Hospital, The Royal Brisbane and Womens' Hospital, and Fremantle Hospital Perth; and from referrals from vascular surgeons and general practitioners in Sydney. Participants will also be recruited from the investigators' registry of participants involved in prior research studies who have agreed to be re-contacted.

People who express an interest in the study will be screened and enrolled by dedicated assessment staff either in person or by telephone. Inclusion criteria are

- Diagnosis of PAD by a vascular specialist, including patient-reported symptoms of IC and/or absence of lower limb pulses and/or resting ankle brachial pressure index (ABPI) $<0.9$ or $>1.4$ [23];

- Able to walk without assistance of another person

- No current or planned involvement in an organised exercise trial or program

- No currently planned peripheral vascular intervention

- Physician confirmed ability to participate in the trial (i.e. medically stable, able to walk and undertake physical activity, attend assessments and comprehend the behavioral counselling). Patients with severe PAD, such as rest pain, arterial ulceration or gangrene requiring urgent vascular intervention will be ineligible.

\section{Exclusion criteria are}

- Previous major lower limb amputation such as above or below knee

- Patients who report doing more than 120 min of moderate-vigorous structured exercise per week.

\section{Sample size calculation}

We hypothesise that over a 4-month period the intervention will increase physical activity by $\sim 50 \%$, evidenced by mean daily step counts of $5000 \pm 3400$ for those receiving the counselling intervention compared to $3300 \pm 2200$ for those randomised to the control group. We also hypothesise that these effects will be maintained at 12 and 24 months post intervention. The effect size and outcome results estimated are based on data from a previous trial [54]. We require 62 participants per group (power $90 \%$, alpha 0.05 , within-subject between visit correlation 0.6) to detect the hypothesised increase in physical activity. Allowing for maintenance of this increase over 24 months, and a drop out of up to $40 \%$, we require 100 participants per group. We therefore will aim to recruit a total of 200 people.

\section{Randomisation}

Eligible and consenting individuals who complete the baseline assessment will be randomly allocated to the control or intervention group. The allocation sequence will be computer-generated using a random number sequence and allocation will occur in block sizes up to 4 and will be stratified based on ABPI $(<0.50,0.50-0.69,0.70-0.89,>1.4)$, study site, and gender. Allocation is concealed by using a central randomisation site, and different project staff for assessment and allocation/intervention delivery. Participants will be blinded to allocation and the investigators' hypotheses. Intervention staff will conduct both intervention and control participant contacts, and therefore cannot be blinded to allocation. They will be instructed not to disclose the allocation status to either the participants or assessment staff.

\section{Study protocol and interventions}

Recruitment and informed consent will be performed by project staff not directly involved in the patients' care. Potential participants will be approached individually either by telephone, mail or in person; provided with the study information; and invited to participate. People who are screened as eligible and provide written consent will be randomised to either the intervention or control group.

An overview of the participant timeframe is presented in Fig. 1. Participants in both groups will receive ongoing medical care from their treating physicians, a written PAD management information sheet including advice to walk, and four individualised contacts from an allied health professional over 3 months (weeks 1, 2, 6 and 12).

The information sheet provides an overview of PAD (symptoms, contributing factors) with recommendations to stop smoking, eat a healthy diet, manage associated conditions such as diabetes and hypertension, and to walk for at least $40 \mathrm{~min}$ three times a week (see Additional file 1). The information includes instruction to walk until a moderate level of pain is experienced in the legs, rest until the pain subsides, and then resume [25].

The allied health professionals are required to be not a qualified psychologist e.g., nurse, dietician, exercise physiologist. They will be trained in the control and intervention protocol by the project psychologist in a face-to-face meeting $(6 \mathrm{~h})$ and given a written manual and summary participant contact protocol. They are required to achieve at least $80 \%$ in a competency-based assessment administered two weeks before commencing participant contact. The assessment requires verbal descriptions of how they would use the study protocol in response to a series of vignettes reflecting participant case scenarios. During the study period, regular individual teleconferences (approximately monthly) will be held between the allied health staff and the psychologist, to discuss and problem-solve participant contact issues that arise. To 


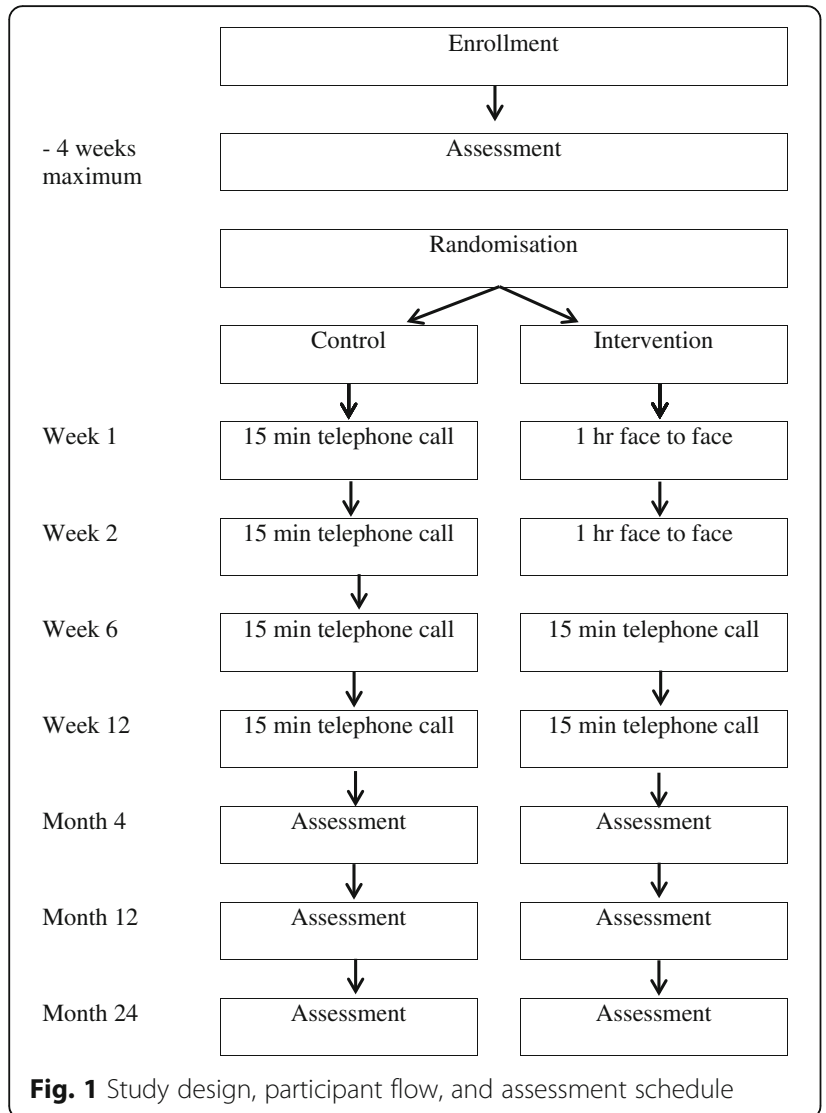

assess intervention fidelity, all participant contacts will be audio recorded and a random selection of sessions (5\%) will be assessed at study conclusion by the psychologist against a protocol checklist.

\section{Passive control group}

Participants in the control group will receive four 15-min telephone calls, during which they will be asked open ended questions about PAD symptoms and general health and wellbeing (e.g., "How have you been since we last spoke?, How does your PAD impact on that?", "How does that affect your PAD?") and receive empathic non-specific responses. Project staff will respond to any specific queries on PAD management or physical activity by encouraging participants to read the written PAD management information sheet and talk with their treating physician. The combination of ongoing medical care from treating physicians and a written PAD management information is intended to represent usual care. The four telephone calls equalises the frequency of participant contacts between the control and intervention group. The telephone calls to control group participants may also mask allocation: participants in a no contact control group could determine allocation to a non-intervention condition.

\section{Active intervention group}

Participants in the intervention group will receive two 1$\mathrm{h}$ face to face behavioral counselling sessions at a university or hospital site, and two 15-min telephone sessions. The counselling protocol used in this trial is based on the 5A Behavioral Counselling Framework, which is an evidence-based approach appropriate for physical activity change in people with chronic conditions and cardiovascular risk [55]. The key components of this approach as applied in this study are:

- Assessment: During the initial session, open ended questions and clarification probes will be used to understand patient perceptions of knowledge of PAD and benefits of walking for PAD, current walking (frequency and duration), and walking change (i.e., risks, barriers, enablers, outcome expectancies, feelings, reasons to change/not change). Subsequent sessions will focus on current walking (frequency and duration), and experiences with walking change (i.e., barriers, enablers, feelings, outcomes).

- Advice: Verbal advice will be provided to acknowledge pros and cons of walking, and confirm understanding and correct misperceptions about PAD and walking (e.g., PAD causes and consequences, interpretation of walking pain, benefits of walking vs. surgery for PAD). Clear and strong advice will be provided on the need to do regular walking to manage PAD symptoms and reduce the risk of serious health events.

- Agree: This will involve collaborative goal setting for walking i.e. graded goal setting (frequency and duration) towards recommendations to do three 40-min sessions per week of interval walking at maximum tolerable speed (i.e., moderate pain level with rest periods).

- Assistance: Discussion on action planning (when, where and with whom to do walking), motivational support (reflecting assessment information), self-regulation strategies, selfmonitoring, social support, and identifying and problem solving barriers to walking (promoting coping, flexibility and control). This information will be individually tailored to participants' interests, opportunities and abilities. The interventionist will provide praise, highlight effort towards achievement and gains, and promote adaptive interpretation of setbacks.

- Arrange: The next contact will be arranged where appropriate.

\section{Participant retention and withdrawal}

Once enrolled, the study site will make every reasonable effort to follow the participant for the entire study period. 
Study site staff are responsible for implementing local standard operating procedures to optimise engagement and minimise attrition. To optimise engagement, contact and assessment times will be prearranged with participants on an individual basis. The allied health professionals will prearrange each contact i.e. the first contact will be organised at the assessment session, the second contact will be organised at the first contact etc. Assessment staff will arrange individualised assessment sessions by telephone.

The allied health professionals will be trained how to respond to participant ambivalence regarding subsequent contacts. The individualised pros and cons of engagement will be acknowledged and problems with engagement discussed. The importance of understanding the diverse spectrum of patient experiences with PAD, irrespective of patient perceptions of change, will be indicated. If participants request to discontinue contact, then participants will be asked if they are willing to engage with the assessment.

Participant non-attendance at face to face sessions or for telephone contacts will be followed up by the allied health professionals. Participant non-attendance at assessment sessions will be followed up by assessment staff. Non-attendance follow up will be primarily by telephone with a minimum of ten attempted contacts conducted at different times of day. Email or a single mail contact may also be attempted. If participants provide a secondary contact at the initial assessment, this will be used to determine if the participant is not available due to extenuating circumstances such as hospitalisation or an adverse event. Participant non-attendance for control/intervention contacts will be rescheduled as soon as possible after originally scheduled contact. If this is not possible within a 1-2 week period, then the contact will be identified as not occurring. The timing of following control/intervention contacts will be relative to the initial session. The assessment timing will remain constant relative to the first intervention/control contact. Participants who do not attend one assessment will be invited to attend subsequent assessment sessions unless they specifically request to withdraw from the study entirely.

Any physician prescribed or participant initiated concomitant care is permitted during the trial. Participants may request to withdraw from the study for any reason at any time. Should participants choose to withdraw, all efforts will be made to identify the reason for withdrawal.

\section{Participant safety}

The behavior counselling intervention in this study is non invasive, low risk and should be well tolerated by the majority of participants. It is therefore unlikely that the trial would require stopping because of safety issues.
Participant safety will be monitored through adverse event reporting via report forms. Adverse events may be identified by assessment or intervention staff during contacts, or spontaneously reported by participants. Definition of adverse events (AE) and serious adverse events (SAE) will be in line with the Good Clinical Practice Guidelines (GCPG) [56]. AEs will be defined as any unfavourable and unintended sign, symptom or disease that occurs in a participant whether it is considered to be study-related or not. A SAE will be defined as an untoward medical occurrence which results in death, requires inpatient hospitalisation or prolongation of existing hospitalisation, results in permanent or significant disability or is life threatening. All SAEs will be reported to the relevant Human Research Ethics Committee (HREC) as soon as possible following identification by study centers. The Trial Steering Committee will monitor SAEs and consider trial termination if events which appear related to the intervention are identified.

\section{Assessment}

Assessments will be conducted at baseline and 4, 12 and 24 months by staff blinded to participant allocation. Each assessment time point will comprise two facility-based visits approximately one week apart, the first for $90 \mathrm{~min}$, and the second for $60 \mathrm{~min}$. At visit 1 (90 $\mathrm{min})$ participants will provide a blood sample and complete physical activity and health-related quality of life questionnaires. The Short Physical Performance Battery (SPPB) and 6-min walk test (6MWT) will be undertaken. An assessment of ABPI will be performed. Smoking status, medical and surgical history, anthropometry, resting heart rate, brachial blood pressure and ambulatory ability will also be assessed. At the end of visit 1 , participants will be fitted with an activPAL $3^{\text {mi }}$ accelerometer, which will be worn for seven consecutive days. At visit 2 (60 $\mathrm{min})$, participants will return the activPAL3 $^{\text {Tw }}$ accelerometer, and complete additional physical activity and health related quality of life questionnaires. At baseline only, the six minute walk test is repeated at visit 2 . Participants will be given the written PAD information sheet at the end of visit 2. Assessments at 4, 12 and 24 months will also include the recording of any study defined clinical events since the previous visit.

\section{Primary outcome measures}

The primary outcome measure will be daily physical activity level or walking (i.e. steps per day). This will be assessed using an activPAL3 ${ }^{\mathrm{mm}}$ accelerometer (PAL Technologies Ltd, Glasgow, United Kingdom) which is a triaxial accelerometer that assesses body position (i.e. sitting/lying and standing) and walking activity from accelerometer recordings at a sampling rate of $20 \mathrm{~Hz}$ and sensitivity of $\pm 2 \mathrm{~g}[57,58]$. The $\operatorname{activPAL}^{\text {ti }}$ unit will be wrapped in a nitrile sleeve, wrapped in waterproof 
dressing and attached to the right thigh of each participant using adhesive waterproof dressings (mid-line; $1 / 3$ of the distance from the inguinal fold to the top of the patella). Recordings will commence at 06:00 on the following morning and continue for seven full days whereupon the activPAL ${ }^{\text {mis }}$ unit will be removed, returned to the investigators and the recording uploaded for analysis. Data are checked to ensure data has been recorded for the period, and if not, participants are asked to redo the assessment. The activPAL ${ }^{\mathrm{mm}}$ has been reported to be reliable and valid in the assessment of walking, body posture, and sedentary behavior during free-living activity $[57,59,60]$ and when used with older adults [61] and patients with IC [62].

\section{Secondary outcome measures}

In addition to the primary measures of daily physical activity level and walking (steps/day), other outcome measures from the activPAL $3^{\text {Tx }}$ will include daily sedentary (sitting/lying) time, number of sit-to-stand transitions, and energy expenditure (total number of kilocalories per day). Other secondary outcome measures using objective assessment will include:

- Ambulatory ability and lower body functioning: This will be assessed using the 6MWT, and the Short Physical Performance Battery (SPPB) which have previously been used as outcome measures in PAD intervention trials $[36,37]$. The 6MWT assesses maximal cardiorespiratory fitness and correlates strongly with physical activity levels among people with PAD, and may therefore reflect walking capacity in everyday life [63]. The SPPB includes three objective tests of lower body function: A hierarchical test of standing balance, a timed 4-m walk and 5timed repetitive chair stands. The SPPB strongly predicts loss of ability to walk $400 \mathrm{~m}$ [40], and is predictive of hospitalisation, nursing home admission and mortality [38, 39, 64]. It is a widely used and valid measure with established test-retest reliability [65].

- Event-based claudication index (EBCI) which is a measure of the ratio of walking bouts to upright time assessed from accelerometer data. Individuals with PAD have a greater EBCI than age matched controls, which indicates a fragmented stop/start walking pattern $[47,62]$.

- Vascular interventions including endovascular and surgical lower limb revascularisations and aneurysm repair.

- Clinical events including cardiovascular events, amputations, vascular and cardiac surgeries/ interventions, musculoskeletal injury, other injuries or illness and death determined from patient reviews and hospital chart reviews, as described elsewhere $[66,67]$.
- Circulating pro-thrombotic markers, proinflammatory cytokines, lipids and RNA expression of circulating cells will be examined at the completion of the trial using stored blood samples obtained at each visit. EDTA and citrate plasma, serum and total blood collected into Paxgene tubes will be obtained at each assessment time. We have previously established methods to assess vascular biomarkers with good repeatability in our central laboratory [68-74].

- Measures of cardiovascular function including heart rate, blood pressure and ABPI assessed with standardised protocols as described elsewhere [75].

- Body composition including waist and hip circumference and body mass index assessed with standardised protocols as described previously [76].

Secondary measures using self-administered questionnaires include the following.

- Physical activity will be assessed using the International Physical Activity Questionnaire (IPAQshort form) and the Physical Activity Scale for the Elderly (PASE). The IPAQ has four item groups that use a past 7 days recall period to ask about frequency (days/week) and time (mins/day) spent walking for at least $10 \mathrm{~min}$ at a time and in vigorous- and moderateintensity activities; and time (mins/day) spent sitting on a week day. The instrument has good repeatability and demonstrated validity comparable with other self-report measures in diverse samples, settings and countries [77]. The PASE has 11 item groups that use a past seven days recall period to ask about frequency (never, 1-2, 3-4, 5-7 days) and average time/day ( $<1 \mathrm{~h},>1-<2 \mathrm{~h}, 2-4 \mathrm{~h},>4 \mathrm{~h}$ ) spent in sitting activities, walking, light sport or recreational activities, moderate sport or recreational activities, strenuous sport or recreational activities, and exercise to increase muscle strength and endurance; participation (yes/no) in domestic activities (light housework, heavy housework, home repairs, yard work, outdoor gardening and caring for another); time (hours/week) spent in volunteer work; and the degree of physical activity done as part of paid or volunteer work (mainly sitting, sitting or standing with some walking, walking with some light-moderate handling, walking and heavy manual work). Additional items will be used to also assess distance (number $\mathrm{km}$ ) of walking and number of flights of stairs to enable Harvard Alumni Questionnaire scoring. The PASE has a good correlation with measures of lower body functioning and the 6MWT [78] and low PASE scores are predictive of cardiac-related mortality after coronary surgery [79].

- Sedentary behavior will be assessed using the SIT-Q$7 \mathrm{~d}$ Questionnaire. This has 18 item groups that use a 
recall of the past seven days to assess average sitting time for meals ( 8 categories ranging from none to $>1 \mathrm{~h} /$ day); for travel (to and from occupation, as part of occupation, and other purposes: 13 categories ranging from 0 to $>7 \mathrm{~h} /$ day); as part of occupation (12 categories ranging from 0 to $>8 \mathrm{~h} /$ day); for nonoccupational screen-based activities (includes reclining for watching television, recreational computer use, playing computer games: 11 categories ranging from 0 to $>8 \mathrm{~h} /$ day); and other activities (reading, household tasks, caring for others, hobbies, socialising, listening to music, other: 11 categories ranging from 0 to $>7 \mathrm{~h} /$ day). Items also assess frequency (times/day) of interrupting sitting time during each of occupation and watching television; the time of going to sleep and getting up and duration of napping; and frequency of consuming each of 11 snack types while watching television (10 categories ranging from none to $>5$ times/day). The questionnaire has acceptable repeatability and good criterion validity for total sedentary time in otherwise healthy people [80].

- Walking ability will be assessed using the Walking Impairment Questionnaire (WIQ). This has 21 items that assess degree of pain and other difficulties (e.g., shortness of breath), difficulty in walking for a range of distances and speeds, and difficulty in climbing stairs. Responses are recorded on a 5-point Likert scale with options of none, slight, some, much, and very/unable. WIQ cut off scores appropriately classify walking performance (absolute claudication distance and peak walking time) assessed by a standardised graded treadmill test in people with PAD [81], and is a valid tool to detect improvement in daily walking ability in people with IC [82].

- Health-related quality of life (HRQoL) will be assessed with the disease-specific Intermittent Claudication Questionnaire (ICQ) [83] which has 16 items that use a recall of the past two weeks and a 5-point Likert scale for responses. Items ask about severity of leg pain (none to very severe); the extent to which leg pain limits a range of activities such as walking and crossing the road (not limited to totally limited); frequency of needing to stop walking (nil to $>3 \mathrm{x} /$ day); and the extent to which the respondent has thought about, felt down about, worried about, had work interference from, had interference with hobbies and social activities from, and had interference doing errands from leg pain (all the time to none of the time). The questionnaire has acceptable test retest reliability, internal consistency and construct validity in people with intermittent claudication [83].

- Health-related quality of life will also be assessed using the PAD Quality of Life (PADQoL), the EQ5D-5 L, and the SF36 (SF-36 v2) tools, which have previously been identified as useful for people with PAD [84-86]. The PADQoL has 38 items that ask about PAD-related issues and experiences such as activity restrictions; feelings of loss, vulnerability, burden, hope, and confidence; and impact on work, family and social activities. Responses are recorded on a 6-point Likert scale with options ranging from strongly agree to strongly disagree. The EQ-5D-5 L is a descriptive system of health-related quality of life states consisting of five dimensions (mobility, self-care, usual activities, pain/discomfort, anxiety/ depression). The responses record five levels of severity (no problems/slight problems/moderate problems/severe problems/extreme problems) within a particular EQ-5D dimension. The SF-36 has 36 items and assesses eight health concepts [87]: limitations in physical activities because of health problems; limitations in social activities because of physical or emotional problems; limitations in usual role activities because of physical health problems; bodily pain; general mental health (psychological distress and well-being); limitations in usual role activities because of emotional problems; vitality (energy and fatigue); and general health perceptions. Two overall component scores can be derived to reflect physical and mental health. Items assess current functioning and during the past four weeks, and responses are provided on Likert type scales with three to five options.

To support an economic evaluation, cost information will also be collected. The following direct health care costs will be determined for every participant using data collected as part of the case report form (CRF):

- The resources associated with the interventions including the costs of training allied health professionals, the cost of delivering the behavior counselling intervention and the cost of monitoring changes in physical activity; These costs include both the salary cost of time spent by staff (pro rata, including on-costs) for the intervention training, implementation, and advisory sessions; administrative costs (e.g., telephone calls), location costs (rooms, utility costs), and travel costs of patients (e.g., time and financial outlay).

- The health services resources used for cardiovascular events will be assessed by data collected on any hospital admissions during followup including reason for admission, length of stay and type of vascular intervention performed. Overall, the cost of each participant's continuous inpatient stay (CIS) will be estimated using the AR-DRG Grouper software licensed by Independent 
Hospital Pricing Authority (IHPA). This software takes into account key variables that impact on a patient's CIS, such as principal and other diagnosis, length of stay, age and sex.

- For each medication prescribed during follow-up the associated costs will be estimated based on the unit costs of prescriptions determined from finance/ purchasing department of the participating hospitals.

Some site-specific assessments will also be conducted (See Additional file 2).

\section{Data management and analyses}

All study information will be stored securely at the study site. All participant information will be stored in locked cabinets in areas with limited access. All data will be identified by a coded identification number only to maintain participant confidentiality. Records that contain names or personal identifiers - such as case notes, consent forms or appointment records - will be stored separately from records identified by code number. Trial documents including study design, protocol, assessment manual, safety reporting forms and case report forms (CRF) and necessary software will be electronically shared with participating study centers.

Data recorded on printed CRFs will be scanned and emailed to the trial coordinating center along with the data file for the activPAL $3^{\text {TM }}$ accelerometer for assessment of data quality and central data entry. Data from the CRFs will be entered into a study database which incorporates data validation rules to reduce transcription errors and can only be accessed by authorised personnel. An extract of the database will be examined regularly and checked by the coordinating centre for data quality. Data entry is done by staff blinded to participant allocation.

Data from the activPAL3 ${ }^{\mathrm{TM}}$ will be analysed using the manufacturer's software (PAL Technologies Ltd, Glasgow, United Kingdom) to derive measures of physical activity and sedentary behavior. Data from the activPAL $3^{\mathrm{TM}}$ will be uploaded and visually inspected for anomalies. Participant data will be considered valid if there is a minimum of $20 \mathrm{~h}$ per day and 5 days of wear time. Default settings of the activPAL3 ${ }^{\text {Ts }}$ will be used to calculate 15-s epochs for each variable using: a minimum sitting and/or upright period of $10 \mathrm{~s}$; a minimum walking cadence of 20 steps per minute; and energy expenditure values of 1.25 MET for sitting/lying, 1.4 MET for quiet standing, and 4 MET for walking cadences of $>119$ steps per minute.

Between group differences in physical activity (primary outcome) and other measures will be analysed using an intention-to-treat approach and repeated measures mixed models. All continuous data will be inspected for normality of distribution visually and statistically, and non-normally distributed data will be mathematically transformed if possible prior to use with parametric statistics. Covariates considered for use in these models include age, gender and any characteristics significantly different between groups at baseline which could potentially confound the variable of interest in each model. Predictors of changes in primary and secondary outcomes will be identified using simple and multiple regression models of relevant baseline characteristics (e.g., BMI and education). The incidence of revascularisation in the intervention and usual care control groups will be compared by chi-square test or logistic regression adjusted for relevant baseline characteristics if needed. Binomial logistic regression will be conducted to identify factors that predict revascularisation. Differences in time to revascularisation will be analysed by Kaplan Meier analysis and $\log$ rank test. Given the low risk of the behavior intervention planned in this study it is highly unlikely that the trial would require stopping and thus no interim analysis is planned. Relative effect sizes and $95 \%$ Confidence Intervals for primary and secondary outcomes will be calculated. A $p$ value of $<0.05$ will be accepted as statistically significant, given that all hypotheses to be tested have been formulated a priori.

The base case economic evaluation will adopt the perspective of the health sector, meaning that the data analysis will be restricted to including the costs and outcomes that directly impact upon the health sector. Two forms of analysis will be undertaken: a cost-effectiveness analysis and a cost-utility analysis. The cost-effectiveness analyses will compare the direct costs of the intervention (minus any cost savings) against the activity outcomes. An incremental cost effectiveness ratio (ICER) will be calculated as follows:

$$
\mathrm{ICER}=(\mathrm{C} 1-\mathrm{C} 0) /(\mathrm{E} 1-\mathrm{E} 0)
$$

where $\mathrm{C} 1$ is the cost of the intervention (minus cost savings); $\mathrm{C} 0$ is the cost of the usual care; $\mathrm{E} 1$ is the effect from the intervention; and E0 is the effect of the usual care. $\mathrm{C} 0$ includes the costs associated with four separate patient appointments, which comprises of a general conversation, and on-going medical care. The difference between $\mathrm{C} 1$ and $\mathrm{C} 0$ is the incremental or additional cost being introduced to the health system. Similarly, the difference between E1 and E0 is the incremental effectiveness of the intervention. Within a sensitivity analysis, any working time lost will be converted into productivity costs, thus widening the perspective of the analysis to include some societal costs.

From the information above, three cost-effectiveness ICERs will be generated, as described below. For each ICER, the incremental cost of the intervention will be identical; whereas for effects, three activity outputs will be used. This analysis will then generate: (i) cost per additional 1000 steps/day walked; (ii) cost per additional 100 calories/day expended; and (iii) cost per additional 
minutes of moderate to vigorous intensity physical activity/day). This analysis will be performed at the 4-month post intervention and 12 and 24 month follow-ups.

The cost-utility analyses will be then be undertaken. The same direct costs will be included as before, however, in this case, the outcome measure will be changed to qualityadjusted life years (QALYs). Health utility scores will be generated for each participant by converting responses to HRQoL questionnaires into a single score using validated algorithms. The algorithm weights responses to HRQoL questions using population preferences and hence health utility score are also known as (preference-weighted) HRQoL. To estimate a quality-adjusted life year for costutility analysis, we will convert the EQ-5D and SF-36 into utility scores (the latter through the SF-6D) [88], and apply Australian utility weights for each [89, 90]. This permits cross-validation checks for the health utility values elicited and exploration of the sensitivity of the incremental outcome to choice of quality of life measure post-intervention. These analyses will be performed at the 12 month and 24 month follow-up. In Australia, a new intervention is generally considered to be cost-effective if the ICER, the mean cost per unitary change in mean utility is $\leq \$ 50,000[91,92]$.

If the intervention is found to successfully improve health outcomes, and to result in reduced time lost from work, then the 'perspective' of the analysis would widen from the health sector to incorporate productivity savings. For those of working age, time lost from employment will be estimated using the World Health Organization's Health and Performance Questionnaire (HPQ) [93]. Working time lost will be converted to productivity losses by using average Gross Domestic Product (GDP) per capita, adjusted pro rata. If individuals are made unemployed (rather than temporary sickness absence) then an assumption will be made regarding the length of time before that position is replaced by another person in the general population. A 90 fractional day period will be assumed, before replacement takes place. Any productivity losses would then be factored into the value of $\mathrm{C} 1$ above (reducing it), and the ICERs would be recalculated. This stage of the analysis would be reported separately as these potential productivity savings do not fall under the health care budget, and so is outside of the health sector funder's perspective.

Uncertainty analysis will be conducted by bootstrapping patient level data and a value of information analysis (VOI) will estimate the need to undertake further research to inform whether the intervention is value for money [94]. All economic evaluation work will be implemented using STATA and Microsoft Excel $^{\circ}$ (Microsoft Corporation, Redmond, WA, USA).

Results will be disseminated via relevant scientific (e.g., journals, conferences), professional (e.g., newsletters), and public (e.g., media) forums. There are no plans to use professional writers.

\section{Discussion}

People with PAD constitute a high-risk population with high rates of cardiovascular events and hospitalization; and greater associated costs compared to patients presenting with coronary artery disease alone [7]. Supervised exercise programs can improve maximal walking distance, pain symptoms and health-related quality of life [20, 28-31], but have limited accessibility [32]. Unsupervised home- or community-based exercise is a potentially attractive alternative to supervised exercise training, and can be effective in increasing walking capacity, community-based walking, and quality of life among people with PAD $[28,95,96]$. Components of successful interventions to promote unsupervised exercise in people with IC include self-monitoring, goal setting, and problem solving barriers [96]. Behavioral counselling typically includes these components, and there is preliminary evidence that brief behavioral counselling by a psychologist can promote sustained increases in walking in people with PAD [53,54], but the generalisability of this to other health professionals is not known. In addition, little is known about the impact of such programs on cardiovascular disease risk [96].

This trial will test the efficacy and cost effectiveness of brief behavioral counselling delivered by allied health professionals in promoting physical activity and improving clinical outcomes in people with PAD. Using a range of allied health professionals to deliver the counselling across several settings will enhance wide scale applicability if it proves effective. This brief intervention could have a significant impact on PAD and how it is managed in clinical settings, and represent a pragmatic means to improve cardiovascular outcomes.

\section{Trial status}

At the time of manuscript submission (October 2016) this study was ongoing. Recruitment and data collection commenced in Townsville in January 2015, and in Brisbane and Sydney in September 2015, and was pending in Perth.

\section{Trial sponsor}

The Queensland Research Centre for Peripheral Vascular Disease; College of Medicine and Dentistry, James Cook University. Townsville QLD 4811, Australia.

\section{Protocol version}

At the time of submission, the trial was using protocol version 5.3 (June 2016). Protocol amendments will be managed and disseminated by the Trial Coordinating Centre.

\section{Additional files}

Additional file 1: PAD Information Sheet. (PDF $240 \mathrm{~kb}$ )

Additional file 2: Site Specific Additional Assessment. (PDF 177 kb) 


\section{Abbreviations}

6MWT: Six minute walk test; ABPI: Ankle brachial pressure index; AE: Adverse events; Cl: Confidence interval; CRF: Case report form; EBCl: Event based claudication index; EDTA: Ethylenediamine tetraacetic acid; FBC: Full blood count; GCPG: Good clinical practice guidelines; GDP: Gross domestic product; HPQ: World Health Organisation Health and Performance Questionnaire; HREC: Human Research Ethics Committee; HRQoL: Health related quality of life; IC: Intermittent claudication; ICER: Incremental cost effectiveness ratio; ICQ: Intermittent Claudication Questionnaire; IPAQ: International Physical Activity Questionnaire; LOS: Length of stay; NCRE-PAD: (Australian) National Research Centre of Excellence in Peripheral Arterial Disease; NHMRC CCRE: (Australian) National Health \& Medical Council Collaborative Centre for Research Excellence; NHMRC: (Australian) National Health \& Medical Council; OR: Odds ratio; PAD: Peripheral arterial disease; PADQoL: Peripheral arterial disease quality of life questionnaire; PPAQ: Paffenbarger Physical Activity Questionnaire; QALY: Quality adjusted life year; RCT: Randomised controlled trial; SAE: Serious adverse events; SPPB: Short physical performance battery; WIQ: Walking Impairment Questionnaire

\section{Acknowledgements}

The authors acknowledge the assistance of Dr Rene Jaeggi as the clinical trials manager for NHMRC funded NCRE-PAD which is overseeing the trial.

\section{Funding}

This project is supported by funding from the (Australian) National Health \& Medical Research Council (NHMRC; APP1063476: Golledge J, Burton NW, Norman P, Fiatarone Singh M, Leicht A, Ademi, Z). This study is also supported by a Centre for Research Excellence grant from the NHMRC (1000967). This funding contributes to costs associated with project staff and participant assessments. These funding bodies have no role in design; the collection, analysis, and interpretation of data; the writing of manuscripts; and the decision to submit the manuscripts for publication.

\section{Availability of data and materials}

The Trial Coordinating Center will oversee any materials sharing processes. Data sharing is not applicable to this article as no datasets were generated or analysed.

\section{Authors' contributions}

$J G$ is the principal investigator and led the study conceptualisation, funding application, and study design and protocol, in collaboration with Phillip Walker, NB, PN, MFS, AL, ZA (grant chief investigators) as well as BP and CR (grant associate investigators). NB designed the counselling intervention, training, and competency assessment; and prepared the manuscript with oversight from JG. LY and SB contributed text on the study and assessment protocol, and data management. KL and RN contributed the economic analyses text. AL contributed the accelerometry text. CR contributed to the sample size, analyses and discussion text. MFS, YM, YD-N and BP contributed text on Sydney site specific details. LY contributed text on the Townsville site specific details. PN contributed site specific text on Perth. All authors contributed to refinement of the study protocol and approved the final manuscript.

\section{Authors' information}

JG has received research grants from the NHMRC, Queensland Government, The Townsville Hospital Private Practice Research Trust and The Heart Foundation. JG holds an NHMRC Practitioner Fellowship (1019921). AL has received research grants from the NHMRC \& The Townsville Hospital Private Practice Research Trust. NB will supervise the allied health interventionists. SB coordinated the trial across sites until July 2016 when JP took over the role. JJ is the lead at, and SR was integral to the establishment of, the Brisbane site. Staff at Townsville: Stuart Best (site coordinator/interventionist until July 2016); Jenna Pinchbeck (interventionist from July 2016); Lisan Yip (assessment/data quality control, site coordinator from July 2016); Cindy Matthews (interventionist from February 2017); Rene Jaeggi (clinical trials manager); Jonathan Golledge (study physician); Ramesh Velu, Nile Allaf and Frank Quigely (recruiting physicians).

Staff at Brisbane: Jason Jenkins (site lead), Jessica Suna (interventionist), Roslyn Clapperton (assessment), Sophie Rowbotham (Associate Investigator); Nicolas Boyne, Danella Favot, Allan Kruger, Murray Ogg, Simon Quinn (recruiting physicians).
Staff at Sydney: Maria Fiatarone Singh (site lead); Belinda Parmenter (co-investigator, recruitment UNSW); Yian Noble (interventionist, recruitment); Yorgi Mavros, Guy Wilson, Matthew Hollings (assessment); Sarah Aitkens, David Robinson (recruiting physicians).

Staff at Perth: Paul Norman (site lead, recruiting physician); Susan Hoskin (interventionist); Wah Wah Lin (assessment).

\section{Competing interests}

The authors declare that they have no competing interests.

\section{Consent for publication}

Not applicable.

\section{Ethics approval and consent to participate}

This study has ethical clearance from The Royal Brisbane and Women's Hospital (ID HREC/14/QRBW/60), The University of Sydney (ID 2015/341), and Fremantle Hospital (ID 14/26), with site specific agreements with The Townsville Hospital (ID SSA/14/QTHS/116), The Royal Brisbane and Women's Hospital (ID SSA/14/QRBW/240) and Fiona Stanley Hospital in Perth (HREC Ref 14-026). All participates will provide written informed consent.

\section{Author details}

'The University of Queensland School of Human Movement \& Nutrition Sciences, St Lucia, Brisbane, QLD 4072, Australia. ${ }^{2}$ University of Basel Institute of Pharmaceutical Medicine, Basel, Switzerland. ${ }^{3}$ University of Monash Department of Epidemiology and Preventive Medicine, Melbourne 3004, VIC, Australia. ${ }^{4}$ Queensland Research Centre for Peripheral Vascular Disease; College of Medicine and Dentistry, James Cook University, Townsville, QLD 4811, Australia. ${ }^{5}$ Exercise, Health and Performance Faculty Research Group, Faculty of Health Sciences, University of Sydney, Sydney, NSW 2141, Australia. ${ }^{6}$ Vascular Surgery The Royal Brisbane and Women's Hospital, Herston, QLD 4059, Australia. ${ }^{7}$ Centre for Health Research, School of Medicine, Western Sydney University, Sydney, NSW 2753, Australia. ${ }^{8}$ Centre for Research Excellence in Chronic Disease Prevention, Australian Institute for Public Health and Tropical Health and Medicine, James Cook University, Townsville, QLD 4811, Australia. ${ }^{9}$ Sport and Exercise Science, College of Healthcare Sciences, James Cook University, Townsville, QLD 4811, Australia. ${ }^{10}$ Exercise, Health and Performance Research Group, Faculty of Health Sciences, University of Sydney, Sydney, NSW 2141, Australia. "'Surgery Fremantle Hospital, The University of Western Australia, Crawley, WA 6009, Australia. ${ }^{12}$ School of Public Health, Curtin University, Perth, WA 6845, Australia. ${ }^{13}$ Department of Exercise Physiology, Faculty of Medicine, University of New South Wales, Sydney, NSW 2052, Australia. ${ }^{14} \mathrm{~S} c h o o l$ of Public Health and Preventive Medicine, Monash University, Melbourne, VIC 3004, Australia. ${ }^{15}$ The University of Queensland School of Medicine, Herston, QLD 4006, Australia. ${ }^{16}$ The Royal Brisbane and Women's Hospital, Herston, QLD 4029, Australia. ${ }^{17}$ Department of Vascular and Endovascular Surgery, The Townsville Hospital, Townsville, QLD 4811, Australia.

Received: 5 October 2016 Accepted: 1 November 2016

Published online: 09 November 2016

\section{References}

1. Golledge J. Lower-limb arterial disease. Lancet. 1997;350:1459-65.

2. Askew C, Parmenter B, Leicht A, Walker P, Golledge J. Exercise \& Sports Science Australia (ESSA) position statement on exercise prescription for patients with peripheral arterial disease and intermittent claudication. J Sci Med Sport. 2013;17(6):623-9.

3. Oka R, Altman M, Giacomini J, Szuba A, Cooke J. Exercise patterns and cardiovascular fitness of patients with peripheral arterial disease. J Vasc Nurs. 2004;22(4):109-14

4. Golomb B, Dang T, Criqui M. Peripheral arterial disease: morbidity and mortality implications. Circulation. 2006;114(7):688-99.

5. McDermott M, Sufit R, Nishida T, Guralnik J, Ferrucci L, Tian L, Liu K, Tan J, Pearce $W$, Schneider J, et al. Lower extremity nerve function in patients with lower extremity ischemia. Arch Intern Med. 2006;166(18):1986-92.

6. Criqui M. Peripheral arterial disease-epidemiological aspects. Vasc Med. 2001;6:3-7.

7. Mahoney E, Wang K, Keo H, Duval S, Smolderen K, Cohen D, Steg G, Bhatt D, Hirsch A, Reduction of Atherothrombosis for Continued Health (REACH) Registry Investigators. Vascular hospitalisation rates and costs in patients 
with peripheral artery disease in the United States. Circ Cardiovasc Qual Outcomes. 2010;3(6):642-51.

8. Long J, Modrall JG, Parker BJ, Swann A, Welborn MB 3rd, Anthony T. Correlation between ankle-brachial index, symptoms and health-related quality of life in patients with peripheral vascular disease. J Vasc Surg. 2004;39(4):723-7.

9. Gardner A, Montgomery P, Killewich L. Natural history of physical function in older men with intermittant claudication. J Vasc Surg. 2004;40:70-8.

10. Regensteiner J, Hiatt W, Coll J, Criqui M, Treat-Jacobson D, McDermott M, Hirsch A. The impact of peripheral arterial disease on health-related quality of life in the Peripheral Arterial Disease Awareness, Risk, and Treatment: New Resources for Survival (PARTNERS) Program. Vasc Med. 2008;13(1):15-24.

11. Smolderen KG, Hoeks SE, Pedersen SS, van Domburg RT, de Liefde II, Poldermans D. Lower-leg symptoms in peripheral arterial disease are associated with anxiety, depression, and anhedonia. Vasc Med. 2009;14(4):297-304.

12. Gardner A, Montgomery P, Parker D. Physical activity is a predictor of all-cause mortality in patients with intermittent claudication. J Vasc Surg. 2008;47(1):117-22.

13. Grenon S, Vittinghoff E, Owens C, Conte M, Whooley M, Cohen B. Peripheral artery disease and risk of cardiovascular events in patients with coronary artery disease: insights from the Heart and Soul Study. Vasc Med. 2013;18(4):176-84.

14. Ankle Brachial Index Collaboration, Fowkes F, Murray G, Butcher I, Heald C, Lee R, Chambless L, Folsom A, Hirsch A, Dramaix M, et al. Ankle brachial index combined with Framingham Risk Score to predict cardiovascular events and mortality: a meta-analysis. JAMA. 2008;300(2):197-208.

15. Selvin E, Erlinger T. Prevalence of and risk factors for peripheral arterial disease in the United States: results from the National Health and Nutrition Examination Survey, 1999-2000. Circulation. 2004;110:738-43.

16. Bergiers S, Vaes B, Degryse J. To screen or not to screen for peripheral arterial disease in subjects aged 80 and over in primary health care: a crosssectional analysis from the BELFRAIL study. BMC Fam Pract. 2011;12:39.

17. Hirsch A, Hartman L, Town R, Virnig B. National health care costs of peripheral arterial disease in the Medicare population. Vasc Med. 2008;13(3):209-15.

18. Medicare Item Reports. 2009. [https://www.medicareaustralia.gov.au/ statistics/mbs_group.shtml].

19. Fowkes F, Leng G. Bypass surgery for chronic lower limb ischaemia. Cochrane Database Syst Rev. 2008;16(2):CD002000.

20. Murphy T, Cutlip D, Regensteiner J, Mohler E, Cohen D, Reynolds M, Massaro J, Lewis B, Cerezo J, Oldenburg N, et al. Supervised exercise versus primary stenting for claudication resulting from aortoiliac peripheral artery disease: six-month outcomes from the claudication: exercise versus endoluminal revascularization (CLEVER) study. Circulation. 2012:125(1):130-9.

21. Network SIG. Diagnosis and management of peripheral arterial disease. A national clinical guideline. Scotland: NHS Quality Improvement; 2006.

22. Layden J, Michaels J, Bermingham S, Higgins B, on behalf of the the Guideline Development Group. Diagnosis and management of lower limb peripheral arterial disease: summary of NICE guidance. Br Med J. 2012;345:e4947.

23. Norgren L, Hiatt W, Dormandy JA, Nehler M, Harris K, Fowkes F, on behalf of the TASC II Working Group. Inter-society consensus for the management of peripheral arterial disease. J Vasc Surg. 2007;45:S5-67.

24. European Stroke Organisation, Tendera M, Aboyans V, Bartelink $M$, Baumgartner I, Clément D, Collet J, Cremonesi A, De Carlo M, Erbel R, et al. ESC guidelines on the diagnosis and treatment of peripheral artery diseases: document covering atherosclerotic disease of extracranial carotid and vertebral, mesenteric, renal, upper and lower extremity arteries: the task force on the diagnosis and treatment of peripheral artery diseases of the European society of cardiology (ESC). Eur Heart J. 2011;32(22):2851-906.

25. Gardner A, Poehlman E. Exercise rehabilitation programs for the treatment of claudication pain. A meta analysis. JAMA. 1995;274(12):975-80.

26. Nordanstig J, Gelin J, Hensäter M, Taft C, Österberg K, Jivegård L. Walking performane and health related quality of life after surgical or endovascular invasive versus non-invasive treatment for intermittent claudication-a prospective randomised trial. Eur J Vasc Endovasc Surg. 2011:42(2):220-7.

27. Mazari F, Kahn J, Carradice D, Samuel N, Abdul Rahman M, Gulati S, Lee H, Mehta T, McCollum P, Chetter I. Randomized clinical trial of percutaneous transluminal angioplasty, supervised exercise and combined treatment for itnermittant claudication due to femoropopliteal arterial disease. Br J Surg. 2012;99(1):39-48.

28. Vemulapalli S, Dolor R, Hasselblad V, Schmit K, Banks A, Heidenfelder B, Patel M, Jones W. Supervised vs unsupervised exercise for intermittent claudication: a systematic review and meta-analysis. Am Heart J. 2015;169(6):924-37.

29. Parmenter BD, Dieberg G, Smart NA. Exercise training for management of peripheral arterial disease: a systematic review and meta-analysis. Sports Med. 2015;45(2):231-44.
30. Lane $\mathrm{R}$, Ellis $\mathrm{B}$, Watson $\mathrm{G}$, Leng $\mathrm{G}$. Exercise for intermittant claudication. Cochrane Database Syst Rev. 2014;18(7):CD000990.

31. Mays R, Rogers R, Hiatt W, Regensteiner J. Community walking programs for treatment of peripheral artery disease. J Vasc Surg. 2013;58(6):1678-87.

32. Crowther R, Spinks W, Leicht A, Sangla K, Quigley F, Golledge J. Effects of a long term exercise program on lower limb mobility, physiological responses, walking performance, and physical activity levels in patients with peripheral arterial disease. J Vasc Surg. 2008;47(2):303-9.

33. Garg P, Liu K, Tian L, Guralnik J, Ferrucci L, Criqui M, Tan J, McDermott M. Physical activity during daily life and functional decline in peripheral arterial disease. Circulation. 2009;119(2):251-60.

34. Garg P, Tian L, Criqui M, Liu K, Ferrucci L, Guralnik J, Tan J, McDermott M. Physical activity during daily life and mortality in patients with with peripheral arterial disease. Circulation. 2006;114:242-8.

35. McDermott M, Liu K, Ferrucci L, Tian L, Guralnik J, Liao Y, Criqui M. Greater sedentary hours and slower walking speed outside the home predict faster declines in functioning and adverse calf muscle changes in peripheral arterial disease. J Am Coll Cardiol. 2011;57(23):2356-64.

36. McDermott M, Ades P, Guralnik J, Dyer A, Ferrucci L, Liu K, Nelson M, LloydJones D, Van Horn L, Garside D, et al. Treadmill exercise and resistance training in patients with peripheral arterial disease with and without intermittent claudication - a randomized controlled trial. JAMA. 2009;301 (2):165-74.

37. Makris G, Lattimer C, Lavida A, Geroulakos G. Availability of supervised exercise programs and the role of structured home-based exercise in peripheral arterial disease. Eur J Vasc Endovasc Surg. 2012;44(6):569-75.

38. Kakkos S, Geroulakos G, Nicolaides A. Improvement of the walking ability in intermittent claudication due to superficial femoral artery occlusion with supervised exercise and pneumatic foot and calf compression: a randomised controlled trial. Eur J Vasc Endovasc Surg. 2005;30(2):164-75.

39. Cheetham D, Burgess L, Ellis M, Williams A, Greenhalgh R, Davies A. Does supervised exercise offer adjuvant benefit over exercise advice alone for the treatment of intermittent claudication? A randomised trial. Eur J Vasc Endovasc Surg. 2004;27(1):17-23.

40. Gelin J, Jivegård L, Taft C, Karlsson J, Sullivan M, Dahllöf A, Sandström R, Arfvidsson B, Lundholm K. Treatment efficacy of intermittent claudication by surgical intervention, supervised physical exercise training compared to no treatment in unselected randomised patients: one year results of functional and physiological improvements. Eur J Vasc Endovasc Surg. 2001;22(2):107-13.

41. Regensteiner J. Exercise rehabilitation for the patients with intermittant claudication: a highly effective yet underutilised treatment. Cardiovasc Hematol Disord Drug Targets. 2004;4(3):233-9.

42. Falcone R, Hirsch A, Regensteiner J, Treat-Jacobson D, Williams M, Hiatt W, Stewart K. Peripheral arterial disease rehabilitation: a review. J Cardpulm Rehabil. 2003;23(3):170-5.

43. Cunningham M, Swanson V, Pappas E, O'Carroll R, Holdsworth R. Illness beliefs and walking behavior after revascularization for intermittent claudication: a qualitative study. J Cardiopulm Rehabil Prev. 2014;34(3):195-201.

44. Cunningham M, Swanson V, Holdsworth R. Using a psychological model to investigate the relationship between illness beliefs and walking behaviour of patients with intermittent claudication. Br J Surg. 2010;97(S1):6.

45. Rejeski W, Tian L, Liao Y, McDermott M. Social cognitive constructs and the promotion of phsyical activity in patients with peripheral artery disease. J Cardiopulm Rehabil Prev. 2008;28:65-72.

46. Gibson J, Kenrick M. Pain and powerlessness: the experience of living with peripheral arterial disease. J Adv Nurs. 1998;27:737-45.

47. Treat-Jacobson D, Halverson S, Ratchford A, Regensteiner J, Lindquist R, Hirsch A. A patient-derived perspective of health-related quality of life with peripheral arterial disease. J Nurs Scholarsh. 2002;34(1):55-60.

48. Lin J, O'Connor E, Evans C, Senger C, Rowland M, Groom H. Behavioral counseling to promote a healthy lifestyle in persons with cardiovascular risk factors: a systematic review for the US Preventive Services Task Force. Ann Intern Med. 2014;161(8):568-73.

49. Kivela K, Elo S, Kyngas H, Kaariainen M. The effects of health coaching on adult patients with chronic diseases: a systematic review. Patient Educ Couns. 2014;97(2):147-57.

50. McDermott M, Liu K, Guralnik J, Criqui M, Spring B, Tian L, Domanchuk K, Ferrucci L, Lloyd-Jones D, Kibbe M, et al. Home based walking exercise intervention in peripheral arterial disease: a randomised clinical trial. JAMA. 2013;310(1):57-65

51. Rejeski W, Spring B, Domanchuk K, Tao HM, Tian L, Zhao LH, McDermott M. A group-mediated, home-based physical activity intervention for patients 
with peripheral artery disease: effects on social and psychological function. J Transl Med. 2014;12:29-29.

52. McDermott M, Guralnik J, Criqui M, Ferrucci L, Zhao L, Liu K, Domanchuk K, Spring B, Tian L, Kibbe M, et al. Home-based walking exercise in peripheral artery disease: 12-month follow-up of the GOALS randomized trial. J Am Heart Assoc. 2014;3(3):e000711.

53. Cunningham M, Swanson V, O'Caroll R, Holdsworth R. Randomized clinical trial of a brief psychological intervention to increase walking in patients with intermittent claudication. Br J Surg. 2012;99(1):49-56.

54. Cunningham M, Swanson V, Holdsworth R, O'Carroll R. Late effects of a brief psychological intervention with intermittent claudication in a randomized clinical trial. Br J Surg. 2013;100(6):756-60.

55. Goldstein M, Whitlock E, DePue J, Planning Committee of the Addressing Multiple Behavioral Risk Factors in Primary Care project. Multiple behavioral risk factor interventions in primary care: summary of research evidence. Am J Prev Med. 2004;27(2):61-79.

56. The Australian Clinical Trial Handbook. A simple, practical guide to the conduct of clinical trials to International standards of Good Clinical Practice (GCP) in the Australian context. Canberra: Commonwealth of Australia; 2006.

57. Ryan C, Grant P, Tigbe W, Granat M. The validity and reliability of a novel activity monitor as a measure of walking. Br J Sports Med. 2006;40(9):779-84

58. PAL Technologies Ltd. ActivPAL3TM operating guide (PALaP3manual.2031. 40525.1.3.docx). Glasgow; PAL Technologies Ltd.; 2010.

59. Grant P, Ryan C, Tigbe W, Granat M. The validation of a novel activity monitor in the measurement of posture and motion during everyday activities. Br J Sports Med. 2006;40(12):992-7.

60. Barreira T, Zderic T, Schuna JJ, Hamilton M, Tudor-Locke C. Free-living activity counts-derived breaks in sedentary time: are they real transitions from sitting to standing? Gait Posture. 2015;42(1):70-2.

61. Grant P, Dall P, Mitchell S, Granat M. Activity-monitor accuracy in measuring step number and cadence in community-dwelling older adults. J Aging Phys Act. 2008;16(2):201-4

62. Clarke C, Holdsworth RJ, Ryan C, Granat M. Free-living physical activity as a novel outcome measure in patients with intermittent claudication. Eur J Vasc Endovasc Surg. 2013;45(2):162-7.

63. McDermott M, Ades P, Dyer AG JM, Kibbe M, Criqui M. Corridor based functional performance measures correlate better with physical activity during daily life than treadmill measures in persons with peripheral arterial disease. J Vasc Surg. 2008;48:1231-7.

64. Bendermacher B, Willigendael E, Teijink J, Prins M. Supervised exercise therapy versus non-supervised exercise therapy for intermittent claudication. Cochrane Database Syst Rev. 2006;19(2):CD005263.

65. van Asselt A, Nicolaï S, Joore M, Prins M, Teijink J, Exercise Therapy in Peripheral Arterial Disease Study Group. Cost-effectiveness of exercise therapy in patients with intermittent claudication: supervised exercise therapy versus a 'go home and walk' advice. Eur J Vasc Endovasc Surg. 2011;41(1):97-103.

66. Golledge J, Ewels C, Muller R, Walker P. Association of chronic kidney disease categories defined with different formulae with major adverse events in patients with peripheral vascular disease. Atherosclerosis. 2014; 232(2):289-97.

67. Golledge J, Quigley F, Velu R, Walker P, Moxon J. Association of impaired fasting glucose, diabetes and their management with the presentation and outcome of peripheral artery disease: a cohort study. Cardiovasc Diabetol. 2014;13:147.

68. Biros E, Gäbel G, Moran C, Schreurs C, Lindeman J, Walker P, Nataatmadja M, West M, Holdt L, Hinterseher I, et al. Differential gene expression in human abdominal aortic aneurysm and aortic occlusive disease. Oncotarget. 2015; 6(15):12984-96.

69. Biros E, Moran C, Wang Y, Walker P, Cardinal J, Golledge J. microRNA profiling in patients with abdominal aortic aneurysms: the significance of miR-155. Clin Sci. 2014;126(11):795-803.

70. Golledge J, Clancy F, Moran C, Biros E, Rush C, Walker P, Norman P. The novel association of the chemokine CCL22 with abdominal aortic aneurysm. Am J Pathol. 2010;176(5):2098-106.

71. Golledge J, Muller R, Clancy P, McCann M, Norman P. Evaluation of the diagnostic and prognostic value of plasma D-dimer for abdominal aortic aneurysm. Eur Heart J. 2011;32(3):354-64.

72. Golledge J, Clancy P, Yeap B, Hankey G, Norman P. Increased serum angiopoietin-2 is associated with abdominal aortic aneurysm prevalence and cardiovascular mortality in older men. Int J Cardiol. 2013;167(4):1159-63.
73. Golledge J, Clancy P, Hankey G, Norman P. Relation between serum thrombospondin-2 and cardiovascular mortality in older men screened for abdominal aortic aneurysm. Am J Cardiol. 2013;111(12):1800-4.

74. Golledge J, Clancy P, Maguire J, Lincz L, Koblar S, McEvoy M, Attia J, Levi C, Sturm J, Almeida $\mathrm{O}$, et al. Plasma angiopoietin-1 is lower after ischemic stroke and associated with major disability but not stroke incidence. Stroke. 2014;45(4):1064-8.

75. Morris D, Cunningham M, Ahimastos A, Kingwell B, Pappas E, Bourke M, Reid C, Stijnen T, Dalman R, Aalami O, et al. TElmisartan in the management of abDominal aortic aneurYsm (TEDY): The study protocol for a randomized controlled trial. Trials. 2015;16:274.

76. Golledge J, Cronin O, lyer V, Bradshaw B, Moxon J, Cunningham M. Body mass index is inversely associated with mortality in patients with peripheral vascular disease. Atherosclerosis. 2013;229(2):549-55.

77. Craig CL, Marshall AL, Sjöström M, Bauman AE, Booth ML, Ainsworth BE, Pratt M, Ekelund U, Yngve A, Sallis JF, et al. International Physical Activity Questionnaire: 12-country reliability and validity. Med Sci Sports Exerc. 2003: 35(8):1381-96

78. Harada N, Chiu V, King A, Stewart A. An evaluation of three self-report physical activity instruments for older adults. Med Sci Sport Exer. 2001;33:962-70.

79. Rengo G, Galasso G, Vitale D, Furgi G, Zincarelli C, Golino L, Femminella G, Piscione F, Rengo F, Leosco D. An active lifestyle prior to coronary surgery is associated with improved survival in elderly patients. J Gerontol A Biol Sci Med Sci. 2010;65(7):758-63.

80. Wijndaele K, De Bourdeaudhuij I, Godino J, Lynch B, Griffin S, Westgate K, Brage S. Reliability and validity of a domain-specific last-7-day sedentary time questionnaire. Med Sci Sports Exerc. 2014;46(6):1248-60.

81. Sagar S, Brown P, Zelt D, Pickett W, Tranmer J. Further clinical validation of the walking impairment questionnaire for classification of walking performance in patients with peripheral artery disease. Int J Vasc Med. 2012; 2012:Article ID 190641

82. Nicolaï SP, Kruidenier LM, Rouwet EV, Graffius K, Prins MH, Teijink JA. The walking impairment questionnaire: an effective tool to assess the effect of treatment in patients with intermittent claudication. J Vasc Surg. 2009;50(1):89-94.

83. Chong P, Garratt A, Golledge J, Greenhalgh R, Davies A. The Intermittent Claudication Questionnaire: a patient-assessed condition-specific health outcome measure. J Vasc Surg. 2002;36(4):764-71.

84. Treat-Jacobson D, Lindquist R, Witt D, Kirk L, Schorr E, Bronas U, Davey C, Regensteiner J. The PADQOL: Development and validation of a PAD-specific quality of life questionnaire. Vasc Med. 2012;17(6):405-15.

85. Leicht A, Crowther R, Muller R, Golledge J. The effects of including quality of life responses in models to predict walking performance of patients with intermittent claudication. Eur J Vasc Endovasc Surg. 2011;41(4):511-7.

86. Beattie D, Golledge J, Greenhalgh R, Davies A. Quality of life assessment in vascular disease: towards a consensus. Eur J Vasc Endovasc Surg. 1997;13(1):13.

87. Ware JJ, Kosinski M, Bjorner J, Turner-Bowker D, Gandek B, Maruish M. User's Manual for the SF-36v2 Health Survey. 2nd ed. Lincoln, Rl: QualityMetric Incorporated; 2007.

88. Brazier J, Roberts J, Deverill M. The estimation of a preference-based index from the SF-36. J Health Econ. 2002;21:217-92.

89. Norman R, Cronin P, Viney R. A pilot discrete choice experiment to explore preferences for EQ-5D-5L health states. Appl Health Econ Health Policy. 2013:11(3):287-98.

90. Norman R, Viney R, Brazier J, Burgess L, Cronin P, King M, Ratcliffe J, Street D. Valuing SF-6D health states using a discrete choice experiment. Med Decis Making. 2013;34(6):773-86.

91. Vos T, Carter R, Barendregt J, Mihalopoulos C, Veerman J, Magnus A, Cobiac L, Bertram M, Wallace A. ACE-Prevention Team: Assessing Cost-Effectiveness in Prevention (ACE-Prevention): Final Report. Brisbane and Deakin University, Melbourne: University of Queensland; 2010.

92. Visconti A, Doyle J, Weir A, Shiell A, Hellard M. Assessing the costeffectiveness of treating chronic hepatitis $C$ virus in people who inject drugs in Australia. J Gastroenterol Hepatol. 2013;28(4):707-16.

93. Kessler R, Barber C, Beck A, Berglund P, Cleany P, McKenas D, Pronk N, Simon G, Stang P, Ustun T, et al. The World Health Organization Health and Work Performance Questionnaire (HPQ). J Occup Environ Med. 2003:45(2):156-74.

94. Briggs A, Weinstein M, Fenwick E, Karnon J, Sculpher M, Palteil AD, on behalf of the ISPOR-SMDM Modeling Good research Practices Task Force. Model parameter estimation and uncertainty analysis. A report of the ISPOR-SMDM Modeling Good Research Practices Task Force Working Group-6. Med Decis Making. 2012;32(5):722-32. 
95. Gardner A, Parker D, Montgomery P, Scott K, Blevins S. Efficacy of quantified home based exercise and supervised exercise in patients with intermittant claudication: a randomized controlled trial. Circulation. 2011;123:491-8.

96. Al-Jundi W, Madbak K, Beard J, Nawaz S, Tew G. Systematic review of homebased exercise programmes for individuals with intermittent claudication. Eur J Vasc Endovasc Surg. 2013;46(6):690-706.

Submit your next manuscript to BioMed Central and we will help you at every step:

- We accept pre-submission inquiries

- Our selector tool helps you to find the most relevant journal

- We provide round the clock customer support

- Convenient online submission

- Thorough peer review

- Inclusion in PubMed and all major indexing services

- Maximum visibility for your research

Submit your manuscript at www.biomedcentral.com/submit 